\title{
Triangle-free induced subgraphs of the unitary polarity graph
}

\author{
Sam Mattheus \\ Francesco Pavese
}

\begin{abstract}
Let $\perp$ be a unitary polarity of a finite projective plane $\pi$ of order $q^{2}$. The unitary polarity graph is the graph with vertex set the points of $\pi$ where two vertices $x$ and $y$ are adjacent if $x \in y^{\perp}$. We show that a triangle-free induced subgraph of the unitary polarity graph of an arbitrary projective plane has at most $\left(q^{4}+q\right) / 2$ vertices. When $\pi$ is the Desarguesian projective plane $\mathrm{PG}\left(2, q^{2}\right)$ and $q$ is even, we show that the upper bound is asymptotically sharp, by providing an example on $q^{4} / 2$ vertices. Finally, the case when $\pi$ is the Figueroa plane is discussed.
\end{abstract}

Keywords: polarity graph, triangle-free, interlacing, Figueroa plane

\section{Introduction}

Let $\pi$ be a finite projective plane of order $q$. A polarity $\perp$ of $\pi$ is an involutory bijective map sending points to lines and lines to points which preserves incidence. A point $x$ of $\pi$ is said to be absolute if $x \in x^{\perp}$. The polarity graph $\mathcal{G}(\pi, \perp)$ is the graph with vertex set the points of $\pi$ where two vertices $x$ and $y$ are adjacent if $x \in y^{\perp}$. Remark that we could have defined the graph $\mathcal{G}(\pi, \perp)$ equivalently with the lines of $\pi$ as vertices. This graph is not simple: every absolute point gives

S. Mattheus: Department of Mathematics, Vrije Universiteit Brussel, Pleinlaan 2, 1050 Brussel, Belgium; e-mail: sam.mattheus@vub.ac.be

F. Pavese: Dipartimento di Meccanica, Matematica e Management, Politecnico di Bari, Via Orabona 4, 70125 Bari, Italy; e-mail: francesco.pavese@poliba.it

Mathematics Subject Classification (2010): Primary 05C50; Secondary 05B25 05C69 05C35

(B) 2018. Licensed under the Creative Commons CC-BY-NC-ND 4.0

http://creativecommons.org/licenses/by-nc-nd/4.0/ 
rise to a loop. A classical theorem by Baer [1] states that every polarity has at least $q+1$ absolute points, which implies that there a polarity graph has at least $q+1$ loops. With a slight abuse of notation we will identify the vertices of the polarity graph with the points (or lines) of the plane. We will say for example that a point $x$ is adjacent to another point $y$. For all definitions and notions regarding projective planes and polarities not mentioned in Section 2, we refer the reader to $[2,21,22]$.

Polarity graphs and their properties have been the subject of study over the last few years. Questions regarding their independence number [15, 26, 27], chromatic number [32] and other properties have been posed and (partially) answered. The motivation behind this line of research lies first of all in the fact that these graphs possess a lot of structure and interesting features. More importantly, polarity graphs are related to some classes of problems in extremal graph theory, among which Ramsey problems and Turán-type problems. For example in the latter, Füredi $[13,14]$ has shown that the unique graph with the most edges among all graphs on $q^{2}+q+1$ vertices not containing $C_{4}$ as a subgraph is the polarity graph, where $\perp$ is an orthogonal polarity, i.e., a polarity with $q+1$ absolute points (which is the least possible as we already mentioned).

Recently, Loucks and Timmons [24] have drawn attention to the following problem.

Question 1.1. What is the largest set of non-absolute vertices in $\mathcal{G}(\pi, \perp)$ inducing a triangle-free subgraph?

Note that only non-absolute vertices are considered, since triangles in a polarity graph cannot contain absolute vertices, see also Section 2.

This problem first appeared in [27] in the context of extremal graph theory, where the authors considered the case when $\pi=\mathrm{PG}(2, q)$ and $\perp$ an orthogonal polarity. They used a construction due to Parsons [28] to obtain an upper bound on the independence number of a 3-uniform hypergraph which first appeared in [23]. Parsons' construction on which they relied is exactly a triangle-free induced subgraph of $\mathcal{G}(\pi, \perp)$.

Loucks and Timmons also mention that one of the motivations behind this question is from Turán-type problems. In particular, we are interested in the maximum number of edges in an $n$-vertex graph without $C_{3}$ or $C_{4}$ as a subgraph. Indeed, it is natural to approach this problem by considering $C_{4}$-free graphs with many edges, and finding a $\mathrm{C}_{3}$-free subgraph thereof.

In this article, we investigate the case when $\perp$ is a unitary polarity. Then the order of the projective plane is necessarily a square, say $q^{2}$, there are $q^{3}+1$ absolute points and the set of absolute points forms a unital $\mathcal{U}$. Note that there are unitals which do not arise from a unitary polarity, see [2] for further results on 
this topic. We denote by $\operatorname{UP}\left(q^{2}\right)$ a unitary polarity graph for an arbitrary projective plane of order $q^{2}$. In the first part of the paper, by refining the techniques used in [24], we obtain the following upper bound for a triangle-free induced subgraph of $\mathrm{UP}\left(q^{2}\right)$.

Theorem 1.2. Let $S$ be a subset of non-absolute vertices of $\operatorname{UP}\left(q^{2}\right)$ inducing a triangle-free subgraph, then

$$
|S| \leq \frac{q^{4}+q}{2}
$$

Moreover, if equality holds and $\ell$ is a line of $\pi$, then $|\ell \cap S| \in\left\{\frac{q^{2}-q}{2}, \frac{q^{2}+q}{2}\right\}$.

In the second part of the paper we deal with the case when $\pi$ is the Desarguesian projective plane $\operatorname{PG}\left(2, q^{2}\right)$. We will denote this graph by $\operatorname{DUP}\left(q^{2}\right)$. When $q$ is even, we are able to show that the upper bound is asymptotically sharp.

Theorem 1.3. For $q$ even, there exists a set of non-absolute vertices of $\operatorname{DUP}\left(q^{2}\right)$ inducing a triangle-free subgraph of size $q^{4} / 2$.

In the last part of the paper we consider the case when $\pi$ is the Figueroa plane $\mathcal{F}$. The plane $\mathcal{F}$ is obtained by the Desarguesian plane $\operatorname{PG}\left(2, q^{3}\right)$, by distorting certain lines. It is known that every polarity of $\mathcal{F}$ induces a polarity of $\mathrm{PG}\left(2, q^{3}\right)[19$, Theorem 4.2]. Vice versa, under certain assumptions, a polarity $\perp$ of the Desarguesian projective plane $\operatorname{PG}\left(2, q^{3}\right)$ gives rise to a polarity $\perp^{\prime}$ of the Figueroa plane $\mathcal{F}$. In this case, we show that a triangle-free induced subgraph of $\mathcal{G}\left(\mathrm{PG}\left(2, q^{3}\right), \perp\right)$ gives rise to a triangle-free induced subgraph of $\mathcal{G}\left(\mathcal{F}, \perp^{\prime}\right)$. This answers a question of Loucks and Timmons [24, Question 1.4] in the case when $\pi$ is the Figueroa plane of order $q^{6}$.

\section{Preliminaries about $\operatorname{UP}\left(q^{2}\right)$}

Before we can prove these results, we need some structural information about $\mathrm{UP}\left(q^{2}\right)$, in particular about the neighbourhood structure, see [22, Chapter XII]. If $x$ is a point of $\pi$, then $x^{\perp}$ denotes its polar line. Suppose first that $x$ is an absolute point, that is, its polar line contains $x$ itself. Therefore, $x$ is adjacent to $q^{2}$ vertices and has a loop. Let $y$ be a neighbour of $x$, then $x^{\perp} \cap y^{\perp}=\{x\}$, which implies that $y$ has no neighbours in $N(x)$. This means that the subgraph induced by $x$ and its neighbours looks like a star. In particular, $x$ can never be contained in a triangle.

On the other hand, if $x$ is a non-absolute point, then it is adjacent to $q^{2}+1$ other vertices. Among these there are $q+1$ absolute points, while the remaining 
$q^{2}-q$ are non-absolute points. Let $y$ be a non-absolute neighbour of $x$, then $x^{\perp}$ and $y^{\perp}$ intersect in a third point $z$. Hence, $x, y, z$ form a triangle in $\operatorname{UP}\left(q^{2}\right)$. Moreover, $z$ is the unique common neighbour of $x$ and $y$. This implies that nonabsolute neighbours of $x$ come in adjacent pairs, giving rise to $\left(q^{2}-q\right) / 2$ triangles with common vertex $x$.

A self-polar triangle of $\pi$ (with respect to $\perp$ ) is a triangle each of whose vertices has the opposite side as polar line. From the discussion above it follows that triangles in $\operatorname{UP}\left(q^{2}\right)$ are in one-to-one correspondence with self-polar triangles of $\pi$ and that there are exactly

$$
\frac{1}{3}\left(q^{4}-q^{3}+q^{2}\right) \frac{q^{2}-q}{2}=\frac{q^{3}\left(q^{2}-q+1\right)(q-1)}{6}
$$

of such triangles. Here and in the sequel we use the term triangle to refer to a triangle in $\mathrm{UP}\left(q^{2}\right)$ or to a self-polar triangle of $\pi$.

\section{The upper bound}

In [24], an upper bound for the number of vertices of $\operatorname{UP}\left(q^{2}\right)$ inducing a trianglefree subgraph was proved. We refine their argument in order to obtain a better upper bound, see Theorem 1.2. To do so, we use techniques from spectral graph theory.

Given two subsets of vertices $S, T$ in a regular graph, let $e(S, T)$ denote the number of ordered pairs $(s, t), s$ adjacent to $t$, where $s \in S, t \in T$. If $S=T$, then we simply write $e(S)$ instead of $e(S, T)$. Note that $e(S, T)=e(T, S)$. The following result, which first appeared in [17, p17 Theorem 2.1.4], is known as the expander mixing lemma and it is a useful tool to estimate $e(S)$. Furthermore, it has found several applications in finite geometry over the last years [3, 25, 27, 31].

Lemma 3.1. Let $G=(V, E)$ be a d-regular simple graph on $n$ vertices with eigenvalues $d=\lambda_{1} \geq \lambda_{2} \geq \cdots \geq \lambda_{n}$. Let $\lambda=\max \left(\left|\lambda_{2}\right|,\left|\lambda_{n}\right|\right)$ be the second largest eigenvalue (in absolute value) and $S \subseteq V$, then the following inequality holds:

$$
\left|2 e(S)-\frac{d|S|^{2}}{n}\right| \leq \lambda|S|\left(1-\frac{|S|}{n}\right) .
$$

We will apply the expander mixing lemma to the graph $\Gamma$ obtained from $\mathrm{UP}\left(q^{2}\right)$ by deleting its $q^{3}+1$ absolute points. Hence, $\Gamma$ is a simple $\left(q^{2}-q\right)$-regular graph on $q^{4}-q^{3}+q^{2}$ vertices. To apply the lemma, the second largest eigenvalue of $\Gamma$ is needed. This can be derived by using a technique due to Haemers, called eigenvalue interlacing. In what follows, we recall some definitions and results from [18]. 
Definition 3.2. Two sequences of real numbers $\lambda_{1} \geq \cdots \geq \lambda_{n}$ and $\mu_{1} \geq \cdots \geq$ $\mu_{m}$ with $m<n$ interlace if

$$
\lambda_{i} \geq \mu_{i} \geq \lambda_{n-m+i} \text { for } 1 \leq i \leq m .
$$

Theorem 3.3. Let $H$ be an induced subgraph of a graph $G$, then the eigenvalues of $H$ interlace those of $G$.

From [15], the eigenvalues of $\operatorname{UP}\left(q^{2}\right)$ are $q^{2}+1, q,-q$ with multiplicities $1,\left(q^{4}+2 q^{2}-q\right) / 2,\left(q^{4}+q\right) / 2$, respectively. Therefore, by Theorem 3.3, the eigenvalues of $\Gamma$, which are $q^{2}-q=\mu_{1} \geq \cdots \geq \mu_{m}$, with $m=q^{4}-q^{3}+q^{2}$, have to satisfy

$$
q=\lambda_{2} \geq \mu_{2} \geq \lambda_{q^{3}+3}=q \text { and } \quad-q=\lambda_{m} \geq \mu_{m} \geq \lambda_{n}=-q .
$$

Either way, the second largest eigenvalue of $\Gamma$ (in absolute value) equals $q$.

Proposition 3.4. Let $S$ be a subset of non-absolute vertices of $\operatorname{UP}\left(q^{2}\right)$ inducing a triangle-free subgraph, then

$$
|S| \leq \frac{q^{4}+q}{2}
$$

Proof. Applying the expander mixing lemma to $\Gamma$, we find

$$
\left|2 e(S)-\frac{\left(q^{2}-q\right)|S|^{2}}{q^{4}-q^{3}+q^{2}}\right| \leq q|S|\left(1-\frac{|S|}{q^{4}-q^{3}+q^{2}}\right) .
$$

Recall that every vertex $x$ is adjacent to $q^{2}-q$ vertices in $\Gamma$, which come in pairs to form $\left(q^{2}-q\right) / 2$ triangles with common vertex $x$. Suppose that $x \in S$, then $x$ can be adjacent to at most one vertex of each triangle. This implies that $x$ has at most $\left(q^{2}-q\right) / 2$ neighbours in $S$ and hence

$$
2 e(S)=\sum_{x \in S} d_{S}(x) \leq \frac{q^{2}-q}{2}|S|,
$$

where $d_{S}(x)$ denotes the number of neighbours in $S$ of a vertex $x$. We can assume that $|S| \geq\left(q^{4}-q^{3}+q^{2}\right) / 2$, otherwise the proposition is vacuously true. Then (1) becomes

$$
\frac{\left(q^{2}-q\right)|S|^{2}}{q^{4}-q^{3}+q^{2}}-q|S|\left(1-\frac{|S|}{q^{4}-q^{3}+q^{2}}\right) \leq 2 e(S) \leq \frac{q^{2}-q}{2}|S| .
$$

Now solving the previous inequality for $|S|$ proves the result. 
The next step is to find out whether the upper bound can be attained. Assume that equality holds. From the proof of Proposition 3.4, we have that each vertex $x \in S$ has degree $d_{S}(x)=\left(q^{2}-q\right) / 2$. From a geometrical point of view, this means that if $x \in S$, then $\left|x^{\perp} \cap S\right|=\left(q^{2}-q\right) / 2$, i.e., a certain number of lines intersect the set $S$ in a constant number of points. By again using eigenvalue interlacing, we will show that an even stronger property holds: for any line $\ell$ we have

$$
|\ell \cap S| \in\left\{\frac{q^{2}-q}{2}, \frac{q^{2}+q}{2}\right\},
$$

i.e. $S$ is a two-intersection set. These point sets have been intensively studied in the literature, see for example $[8,29]$ and references therein.

Definition 3.5. The interlacing of two sequences of real numbers $\lambda_{1} \geq \cdots \geq \lambda_{n}$ and $\mu_{1} \geq \cdots \geq \mu_{m}, m<n$, is tight if there exists $0 \leq k \leq m$ such that

$$
\lambda_{i}=\mu_{i} \text { for } 1 \leq i \leq k \text { and } \lambda_{n-m+i}=\mu_{i} \text { for } k+1 \leq i \leq m .
$$

Theorem 3.6. [18, p596 Corollary 2.3] Let $A$ be a symmetric $n \times n$ matrix partitioned as

$$
A=\left(\begin{array}{ccc}
A_{1,1} & \ldots & A_{1, m} \\
\vdots & & \vdots \\
A_{m, 1} & \ldots & A_{m, m}
\end{array}\right),
$$

such that $A_{i, i}$ is a square matrix for all $1 \leq i \leq m$. The quotient matrix $B$ is the $m \times m$ matrix with entries the average row sums of the blocks of $A$. More precisely,

$$
B=\left(b_{i, j}\right), b_{i, j}=\frac{1}{n_{i}} \mathbf{1}^{t} A_{i, j} \mathbf{1}
$$

where 1 denotes the all one column vector and $n_{i}$ is the number of rows of $A_{i, j}$. Then the following holds

1. The eigenvalues of $B$ interlace those of $A$;

2. if the interlacing is tight, then $A_{i, j}$ has constant row and column sums for $1 \leq i, j \leq m$.

Lemma 3.7. Let $S$ be a subset of non-absolute vertices of $\mathrm{UP}\left(q^{2}\right)$ inducing a trianglefree subgraph, with $|S|=\left(q^{4}+q\right) / 2$. Then, if $x$ is a point of $\pi$, we have that

$$
\left|x^{\perp} \cap S\right|= \begin{cases}\frac{q^{2}-q}{2} & \text { if } x \in S, \\ \frac{q^{2}+q}{2} & \text { if } x \notin S .\end{cases}
$$


Proof. Let $A$ be the adjacency matrix of $\operatorname{UP}\left(q^{2}\right)$. Recall that $\operatorname{UP}\left(q^{2}\right)$ has loops, and the diagonal entries of $A$ are ones. We can partition the points of $\pi$ into three sets: the set of absolute points $\mathcal{U}$, the set of interest $S$ and their complement $R$. Hence $|R|=q(q-1)\left(q^{2}-q+1\right) / 2$. By considering the above partition, after reordering rows and columns, we get

$$
A=\left(\begin{array}{lll}
A_{1,1} & A_{1,2} & A_{1,3} \\
A_{2,1} & A_{2,2} & A_{2,3} \\
A_{3,1} & A_{3,2} & A_{3,3}
\end{array}\right) .
$$

Consider the quotient matrix

$$
B=\left(\begin{array}{ccc}
|\mathcal{U}|^{-1} 2 e(\mathcal{U}) & |\mathcal{U}|^{-1} e(\mathcal{U}, S) & |\mathcal{U}|^{-1} e(\mathcal{U}, R) \\
|S|^{-1} e(S, \mathcal{U}) & |S|^{-1} 2 e(S) & |S|^{-1} e(S, R) \\
|R|^{-1} e(R, \mathcal{U}) & |R|^{-1} e(R, S) & |R|^{-1} 2 e(R)
\end{array}\right)
$$

We already know a few entries. Indeed, by hypothesis, $|S|^{-1} 2 e(S)=\left(q^{2}-q\right) / 2$. Moreover, every absolute point is adjacent with exactly one absolute point (itself), so $|\mathcal{U}|^{-1} 2 e(\mathcal{U})=1$ and every non-absolute point is adjacent with $q+1$ absolute points, hence $|S|^{-1} e(S, \mathcal{U})=|R|^{-1} e(R, \mathcal{U})=q+1$. Analogously, since every point of $S$ is adjacent with exactly $\left(q^{2}-q\right) / 2$ points of $R$ we get $|S|^{-1} e(S, R)=$ $\left(q^{2}-q\right) / 2$. As $e(\mathcal{U}, S)=e(S, \mathcal{U})$, it follows that $|\mathcal{U}|^{-1} e(\mathcal{U}, S)=\left(q^{2}+q\right) / 2$, and similarly $|\mathcal{U}|^{-1} e(\mathcal{U}, R)=\left(q^{2}-q\right) / 2$ and $|R|^{-1} e(R, S)=\left(q^{2}+q\right) / 2$. Lastly, since the sum of the elements of a row of $A$ equals $q^{2}+1$, we obtain $|R|^{-1} 2 e(R)=$ $\left(q^{2}-3 q\right) / 2$. Collecting these values gives

$$
B=\left(\begin{array}{ccc}
1 & \frac{q^{2}+q}{2} & \frac{q^{2}-q}{2} \\
q+1 & \frac{q^{2}-q}{2} & \frac{q^{2}-q}{2} \\
q+1 & \frac{q^{2}+q}{2} & \frac{q^{2}-3 q}{2}
\end{array}\right) .
$$

The eigenvalues of $B$ are $q^{2}+1,-q,-q$, which shows that the interlacing is tight. By Theorem 3.6, every block $A_{i, j}$ has constant row sum and constant column sum. This means that every vertex in $\mathcal{U}$ or $R$ is adjacent to precisely $\left(q^{2}+q\right) / 2$ vertices in $S$.

Remark 3.8. Let $S$ be a subset of non-absolute vertices of $\operatorname{UP}\left(q^{2}\right)$ inducing a triangle-free subgraph, with $|S|=\left(q^{4}+q\right) / 2$. Then, in the language of [10], we have that $S$ is an intriguing set of $\Gamma$, which could also be shown using Proposition 3.8 of that article.

Remark 3.9. Let $S$ be a subset of non-absolute vertices of $\operatorname{UP}\left(q^{2}\right)$ inducing a triangle-free subgraph such that $|S|=\left(q^{4}+q\right) / 2$. Then the set $S \cup \mathcal{U}$ is a 
two-intersection set. Indeed, if $x \in S$, then $\left|x^{\perp} \cap S\right|=\left(q^{2}-q\right) / 2$ and hence $\left|x^{\perp} \cap(S \cup \mathcal{U})\right|=\left(q^{2}+q\right) / 2+1$. On the other hand, if $x \notin S$, then $\left|x^{\perp} \cap S\right|=$ $\left(q^{2}+q\right) / 2$ and hence $\left|x^{\perp} \cap(S \cup \mathcal{U})\right|$ equals either $\left(q^{2}+q\right) / 2+1$ or $\left(q^{2}+q\right) / 2+q+1$, according as $x \in \mathcal{U}$ or $x \notin \mathcal{U}$. It follows that $S \cup \mathcal{U}$ is a set of $(q+2)\left(q^{3}+1\right) / 2$ points such that every line meets $S \cup \mathcal{U}$ is either $\left(q^{2}+q+2\right) / 2$ or $\left(q^{2}+3 q+2\right) / 2$ points. Since no such a set exists in $\mathrm{PG}(2,4)$ or in $\mathrm{PG}(2,9)$, see [29], it follows that in these cases the upper bound of Proposition 3.4 cannot be attained.

\section{The Desarguesian plane}

Let $\pi$ be the Desarguesian projective plane $\mathrm{PG}\left(2, q^{2}\right)$, with $q=p^{h}, p$ a prime, $h$ a positive integer. The set of absolute points of a unitary polarity of $\operatorname{PG}\left(2, q^{2}\right)$ is called a Hermitian curve. In this case, if $q$ is even, by means of constructive arguments, we are able to show a lower bound close to the upper bound of Theorem 1.2. In particular we will prove the existence of a triangle-free subgraph of $\operatorname{DUP}\left(q^{2}\right)$ having $q^{4} / 2$ vertices, see Theorem 1.3. The strategy is the following: we will fix a unitary polarity $\perp$ and hence a Hermitian curve $\mathcal{U}$; we will consider a set $\mathcal{P}$ containing $q$ Hermitian curves such that $\mathcal{U}$ belongs to $\mathcal{P}$ and elements in $\mathcal{P}$ pairwise intersect at a common point. Then we will select $q / 2$ Hermitian curves in $\mathcal{P} \backslash\{\mathcal{U}\}$ and show that the set of points covered by these Hermitian curves distinct from their common point possesses the required properties.

\subsection{A lower bound}

The projective plane $\mathrm{PG}\left(2, q^{2}\right)$ will be represented via homogeneous coordinates over the Galois field $\mathbb{F}_{q}$, i.e., represent the points of $\operatorname{PG}\left(2, q^{2}\right)$ by $\langle(x, y, z)\rangle$, $x, y, z \in \mathbb{F}_{q},(x, y, z) \neq(0,0,0)$, and similarly lines by $\langle[a, b, c]\rangle, a, b, c \in \mathbb{F}_{q}$, $[a, b, c] \neq[0,0,0]$. Incidence is given by $a x+b y+c z=0$. To avoid awkward notation the angle brackets will be dropped in what follows. The group consisting of all projectivities of $\operatorname{PG}\left(2, q^{2}\right)$ is denoted by $\operatorname{PGL}\left(3, q^{2}\right)$. The point $U_{i}$ is the point with 1 in the $i$-th position and 0 elsewhere. As any two Hermitian curves are projectively equivalent [21, Chapter 5], we may assume that $\mathcal{U}$ has equation

$$
X^{q} Y+X Y^{q}+Z^{q+1}=0
$$

In other words, the matrix defining the polarity is the matrix

$$
P=\left(\begin{array}{lll}
0 & 1 & 0 \\
1 & 0 & 0 \\
0 & 0 & 1
\end{array}\right)
$$


two vertices $\left(x_{1}, y_{1}, z_{1}\right)$ and $\left(x_{2}, y_{2}, z_{2}\right)$ in $\operatorname{DUP}\left(q^{2}\right)$ are adjacent if and only if

$$
\left(\begin{array}{lll}
x_{1} & y_{1} & z_{1}
\end{array}\right)\left(\begin{array}{lll}
0 & 1 & 0 \\
1 & 0 & 0 \\
0 & 0 & 1
\end{array}\right)\left(\begin{array}{c}
x_{2}^{q} \\
y_{2}^{q} \\
z_{2}^{q}
\end{array}\right)=0
$$

and

$$
(x, y, z)^{\perp}=\left[y^{q}, x^{q}, z^{q}\right] .
$$

Let $G \cong \operatorname{PGU}\left(3, q^{2}\right)$ be the subgroup of $\operatorname{PGL}\left(3, q^{2}\right)$ leaving $\mathcal{U}$ invariant. We shall find it helpful to work with the elements of $\operatorname{PGL}\left(3, q^{2}\right)$ as matrices in $\operatorname{GL}\left(3, q^{2}\right)$ and the points of $\operatorname{PG}\left(2, q^{2}\right)$ as column vectors, with matrices acting on the left.

The point $U_{2}$ clearly belongs to $\mathcal{U}$ and its polar line $U_{2}^{\perp}$ is the line $\ell: X=0$. A pencil of Hermitian curves of $\operatorname{PG}\left(2, q^{2}\right)$ generated by the Hermitian curves $\mathcal{H}, \mathcal{H}^{\prime}$ with equations $F=0$ and $F^{\prime}=0$, respectively, is the set of unitals defined by $\alpha F+\beta F^{\prime}=0$, where $\alpha, \beta \in \mathbb{F}_{q},(\alpha, \beta) \neq(0,0)$. We can consider the pencil $\mathcal{P}$ generated by $\mathcal{U}$ and the degenerate Hermitian curve $\ell$ defined by $X^{q+1}=0$. After normalization, this pencil contains the $q$ Hermitian curves defined by

$$
\mathcal{U}_{\lambda}: \lambda X^{q+1}+X^{q} Y+X Y^{q}+Z^{q+1}=0,
$$

where $\lambda \in \mathbb{F}_{q}$. Clearly, when $\lambda=0$ we see that $\mathcal{U}_{0}=\mathcal{U}$, while for " $\lambda=\infty$ " we retrieve the line $\ell$, which will be of lesser importance. Every point not on the line $\ell$ belongs to exactly one Hermitian curve of $\mathcal{P}$, while the point $U_{2}$ is common to all Hermitian curves in $\mathcal{P}$.

Lemma 4.1. There exists a subgroup $K$ of $G$ of order $q^{3}$ acting regularly on points of $\mathcal{U}_{\lambda} \backslash\left\{U_{2}\right\}, \lambda \in \mathbb{F}_{q}$.

Proof. Here, we shall consider the points of $\operatorname{PG}\left(2, q^{2}\right)$ as column vectors, with matrices acting on the left. Let $K$ be the subgroup of $G$ whose elements are associated with the following matrices

$$
\left(\begin{array}{ccc}
1 & 0 & 0 \\
a & 1 & -b^{q} \\
b & 0 & 1
\end{array}\right)
$$

where $(1, a, b) \in \mathcal{U}$. Then $K$ is a group of order $q^{3}$. Straightforward calculations show that if $P \in \mathcal{U}_{\lambda}$ and $g \in K$, then $P^{g} \in \mathcal{U}_{\lambda}$ and that the stabilizer in $K$ of a point $P \in \mathcal{U}_{\lambda} \backslash\left\{U_{2}\right\}$ is trivial.

Proposition 4.2. Let $\lambda_{1}, \lambda_{2}, \lambda_{3} \in \mathbb{F}_{q} \backslash\{0\}$, not necessarily distinct. If $\mathcal{U}_{\lambda_{1}} \cup \mathcal{U}_{\lambda_{2}} \cup$ $\mathcal{U}_{\lambda_{3}}$ contains a triangle, not containing the common absolute point $U_{2}$, then

$$
\lambda_{1} \lambda_{2}+\lambda_{2} \lambda_{3}+\lambda_{1} \lambda_{3}=0
$$


Proof. Suppose that we do have three points $P, Q, R$ forming a triangle and contained in $\mathcal{U}_{\lambda_{1}} \cup \mathcal{U}_{\lambda_{2}} \cup \mathcal{U}_{\lambda_{3}}$. Taking into account Lemma 4.1, we may assume that $P$ is the point $\left(1, x_{1}, 0\right) \in \mathcal{U}_{\lambda_{1}}$, where

$$
\lambda_{1}+x_{1}+x_{1}^{q}=0
$$

The second point $Q=\left(1, x_{2}, y_{2}\right) \in \mathcal{U}_{\lambda_{2}}$ has to be on the line $P^{\perp}: x_{1}^{q} X+Y=0$, which implies that $x_{2}=-x_{1}^{q}$. Here we find by (3) that

$$
\lambda_{2}-x_{1}^{q}-x_{1}+y_{2}^{q+1}=\lambda_{1}+\lambda_{2}+y_{2}^{q+1}=0 .
$$

For the third point $R$, we find in the same way that it should be of the form $\left(1,-x_{1}^{q}, y_{3}\right) \in \mathcal{U}_{\lambda_{3}}$, where

$$
\lambda_{1}+\lambda_{3}+y_{3}^{q+1}=0 .
$$

Moreover, $R \in Q^{\perp}$, where $Q^{\perp}:-x_{1} X+Y+y_{2}^{q} Z=0$. This implies that

$$
-x_{1}-x_{1}^{q}+y_{2}^{q} y_{3}=\lambda_{1}+y_{2}^{q} y_{3}=0 .
$$

Remark that $y_{2}$ nor $y_{3}$ can equal zero, for otherwise one of $Q$ or $R$ would have to be $U_{2}$. Multiplying this last equation by $y_{2} y_{3}^{q}$ and using (4), (5), (6), we obtain that

$$
\begin{aligned}
& \lambda_{1} y_{2} y_{3}^{q}+y_{2}^{q+1} y_{3}^{q+1}=\lambda_{1}\left(-\lambda_{1}^{q}\right)+\left(-\lambda_{1}-\lambda_{2}\right)\left(-\lambda_{1}-\lambda_{3}\right)= \\
&=-\lambda_{1}^{2}+\lambda_{1}^{2}+\lambda_{1} \lambda_{2}+\lambda_{2} \lambda_{3}+\lambda_{1} \lambda_{3}=\lambda_{1} \lambda_{2}+\lambda_{2} \lambda_{3}+\lambda_{1} \lambda_{3}=0,
\end{aligned}
$$

as $\lambda_{1} \in \mathbb{F}_{q}$.

Therefore, if we can find a set $\Lambda \subseteq \mathbb{F}_{q} \backslash\{0\}$ such that for every $\lambda_{1}, \lambda_{2}, \lambda_{3} \in \Lambda$ equation (2) is never satisfied, then the set $\bigcup_{\lambda \in \Lambda} \mathcal{U}_{\lambda} \backslash\left\{U_{2}\right\}$ induces a triangle-free subgraph on $|\Lambda| q^{3}$ vertices. We will call such a set $\Lambda$ a good set.

Remark 4.3. Note that, although Proposition 4.2 is useless in the case when $p=3$, in all the other cases it provides the (weak) lower bound $q^{3}$ for the number of vertices of a triangle-free induced subgraph of $\operatorname{DUP}\left(q^{2}\right)$. This can be achieved by considering the points of the unital $\mathcal{U}_{\lambda} \backslash\left\{U_{2}\right\}, \lambda \neq 0$.

In order to find a good set $\Lambda$ of large size, we first restate the problem.

Lemma 4.4. $A$ good set $\Lambda$ is equivalent to a set $\bar{\Lambda} \subseteq \mathbb{F}_{q} \backslash\{0\}$ such that the sum of any three (not necessarily distinct) of its elements is never zero. In particular $|\Lambda|=|\bar{\Lambda}|$. 
Proof. Let $x, y, z \in \Lambda$. Then $x y+x z+y x \neq 0$ and dividing by $x y z$ we have that $x^{-1}+y^{-1}+z^{-1} \neq 0$. Let $\bar{\Lambda}:=\left\{x^{-1} \mid x \in \Lambda\right\}$. Then the sum of any three (not necessarily distinct) elements of $\bar{\Lambda}$ is never zero. Vice versa, let $a, b, c \in \bar{\Lambda}$. Then $a+b+c \neq 0$ and dividing by $a b c$ we have that $b^{-1} c^{-1}+a^{-1} c^{-1}+a^{-1} b^{-1} \neq 0$. If $\Lambda:=\left\{x^{-1} \mid x \in \bar{\Lambda}\right\}$, then for any three (not necessarily distinct) elements of $\Lambda,(2)$ is never satisfied.

We split into two cases, depending on the parity of $q$.

Lemma 4.5. If $q$ is even, then there exists a good set $\Lambda \subseteq \mathbb{F}_{q} \backslash\{0\}$ of size $q / 2$.

Proof. First we show the existence of an additive subgroup $H$ of size $q / 2$ such that $1 \notin H$. Consider $\mathbb{F}_{q}$ as a vector space $V$ over $\mathbb{F}_{2}$, then the additive subgroups of $\mathbb{F}_{q}$ are in one-to-one correspondence with subspaces of this vector space $V$. In particular, a subgroup of size $q / 2$ corresponds to a hyperplane of $V$. It is immediate that any of the $q / 2$ hyperplanes not through the vector corresponding to 1 , gives rise to a subgroup $H$ satisfying all conditions.

Then we define the set $\bar{\Lambda}$ by

$$
\bar{\Lambda}=\{h+1 \mid h \in H\} .
$$

It is clear that sum of any three (not necessarily distinct) elements is never zero as

$$
(a+1)+(b+1)+(c+1)=0
$$

is equivalent with

$$
a+b+c=1,
$$

in contradiction with the choice of $H$. Therefore, we find by Lemma 4.4 that $\Lambda=\left\{x^{-1} \mid x \in \bar{\Lambda}\right\}$ is a good set of size $q / 2$.

This shows that for $q$ even, we have a triangle-free induced subgraph of $\operatorname{DUP}\left(q^{2}\right)$ of size $q^{4} / 2$, which proves Theorem 1.3 .

For $q$ odd, the situation is very different.

Remark 4.6. If $q$ is odd, let $P$ be a point of $\mathrm{PG}\left(2, q^{2}\right)$ not in $\mathcal{U}$ and let $T_{P}$ be the set of non-absolute points distinct from $P$ lying on the $q+1$ lines containing $P$ and tangent to $\mathcal{U}$. Then $\left|T_{P}\right|=\left(q^{2}-1\right)(q+1)$. We claim that $T_{P}$ contains no triangle. Otherwise, if $Q_{1}, Q_{2}, Q_{3}$ were a triangle contained in $\mathcal{U}$, we would obtain a configuration consisting of seven points: $P, Q_{1}, Q_{2}, Q_{3}, P Q_{1} \cap \mathcal{U}, P Q_{2} \cap$ $\mathcal{U}, P Q_{3} \cap \mathcal{U}$ and seven lines $P^{\perp}, Q_{1}^{\perp}, Q_{2}^{\perp}, Q_{3}^{\perp}, P Q_{1}, P Q_{2}, P Q_{3}$ such that through each point there pass three lines and each line contains three points, i.e., a Fano plane $\mathrm{PG}(2,2)$. On the other hand, if $q$ is odd, $\mathrm{PG}(2,2)$ cannot be embedded in 
$\mathrm{PG}\left(2, q^{2}\right)$. Note that a larger set containing $T_{P}$ and not containing triangles can be obtained by adding to $T_{P}$ the $q^{2}-q$ non-absolute points on the line $P^{\perp}$. This gives the (weak) lower bound $q^{3}+2 q^{2}-2 q-1$ for the number of vertices of a triangle-free induced subgraph of $\operatorname{DUP}\left(q^{2}\right)$, in the case when $q$ is odd.

A better lower bound than that of Remark 4.6 can be obtained in the case when $q$ is odd and $p \neq 3$. It relies on the following results, whose proofs were communicated to us by Bence Csajbók [9] and by Anurag Bishnoi and Aditya Potukuchi [4] independently for the prime case.

Theorem 4.7. Let $q=p^{h}$ be odd, $3 \neq p=3 k \pm 1$. Then there exists a good set $\Lambda \subseteq \mathbb{F}_{q} \backslash\{0\}$ of size $k q / p$.

Proof. By Lemma 4.4 it is sufficient to show the existence of a set $\bar{\Lambda} \subseteq \mathbb{F}_{q} \backslash\{0\}$ such that the sum of any three (not necessarily distinct) of its elements is never zero. Assume first that $h=1$. If $p=3 k+1$, then the sum of any three (not necessarily distinct) elements of $\bar{\Lambda}=\{1,2,3, \ldots, k\}$ is non-zero. If $p=3 k-1$, then the sum of any three (not necessarily distinct) elements of $\bar{\Lambda}=\{k, k+$ $1, \ldots, 2 k-1\}$ is non-zero.

Assume now that $h>1$. Consider again $\mathbb{F}_{q}$ as a vector space $V$ over $\mathbb{F}_{p}$, then the additive subgroups of $\mathbb{F}_{q}$ are in one-to-one correspondence with subspaces of this vector space $V$. Let $A$ be any hyperplane of $V$ which does not contain 1. Hence $A \cap \mathbb{F}_{p}=\{0\}$. Also, let $\bar{\Lambda}^{\prime}$ be a subset of $\mathbb{F}_{p}$ of size $k$ such that the sum of any three (not necessarily distinct) of its elements is never zero. Put $\bar{\Lambda}=$ $A+\bar{\Lambda}^{\prime}:=\{a+\lambda \mid a \in A, \lambda \in \bar{\Lambda}\}$. We claim that $\bar{\Lambda}$ has the required property. Indeed, if we had $\left(a_{1}+\lambda_{1}\right)+\left(a_{2}+\lambda_{2}\right)+\left(a_{3}+\lambda_{3}\right)=0$, for some $a_{i} \in A$ and $\lambda_{i} \in \bar{\Lambda}^{\prime}, 1 \leq i \leq 3$, then $-\left(a_{1}+a_{2}+a_{3}\right)=\lambda_{1}+\lambda_{2}+\lambda_{3} \in \mathbb{F}_{p}$. However, $A \cap \mathbb{F}_{p}=\{0\}$, and thus $\lambda_{1}+\lambda_{2}+\lambda_{3}=0$, contradicting the choice of $\bar{\Lambda}^{\prime}$.

This good set for $q$ odd provides a triangle-free induced subgraph of size $q^{4} / 3+o\left(q^{4}\right)$, which does not match the upper bound asymptotically.

\subsection{Properties of the graph}

Assume that $q$ is even and let $\Sigma$ be a triangle-free induced subgraph of $\operatorname{DUP}\left(q^{2}\right)$ on $q^{4} / 2$ vertices constructed in subsection 4.1. Here, we investigate further properties of the graph $\Sigma$. To start off, we prove that $\Sigma$ is regular.

Proposition 4.8. The graph $\Sigma$ is $q(q-1) / 2$-regular.

Proof. Let $P$ be a point of $\operatorname{PG}\left(2, q^{2}\right), P \notin \ell$, where $\ell$ is the tangent to $\mathcal{U}$ at $U_{2}$. As we have partitioned all points of $\mathrm{PG}\left(2, q^{2}\right)$ into the union of the $q$ sets $\mathcal{U}_{\lambda} \backslash\left\{U_{2}\right\}$ 
and the line $\ell$, it is easy to see that the line $P^{\perp}$ contains a point of $\ell$, is secant to $q-1$ Hermitian curves of $\mathcal{P}$ and is tangent to exactly one Hermitian curve of $\mathcal{P}$. Consider a vertex $v \in \mathcal{U}_{\lambda} \backslash\left\{U_{2}\right\}, \lambda \in \Lambda$ and let $\mathcal{U}_{\mu}$ be the unique Hermitian curve of $\mathcal{P}$ such that $\left|v^{\perp} \cap \mathcal{U}_{\mu}\right|=1$. Taking into account Lemma 4.1, we can assume that the point $v$ has coordinates $(1, x, 0)$, where $x+x^{q}=\lambda$. Then $v^{\perp}$ has dual coordinates $\left[x^{q}, 1,0\right]$ and we have to find $\mu$ such that $v^{\perp}$ is tangent to $\mathcal{U}_{\mu}$. This means finding $\mu$ such that

$$
0=\mu X^{q+1}+\left(x+x^{q}\right) X^{q+1}+Z^{q+1}=\mu X^{q+1}+\lambda X^{q+1}+Z^{q+1}
$$

has only one solution. Since $q$ is even, it follows immediately that $\lambda=\mu$. Hence, every vertex $v \in \mathcal{U}_{\lambda}$ has exactly one neighbour in $\mathcal{U}_{\lambda}$. Moreover, it has $q+1$ neighbours in $\Sigma$ on the $q / 2-1$ other Hermitian curves $\mathcal{U}_{\lambda}, \lambda \in \Lambda \backslash\{\mu\}$, which implies that the degree in $\Sigma$ of every vertex of $\Sigma$ is $q(q-1) / 2$.

In fact, with a similar proof, one can show the exact intersection numbers for any line with $S$.

Corollary 4.9. Let $x$ be a point of $\mathrm{PG}\left(2, q^{2}\right)$, then

$$
\left|x^{\perp} \cap \Sigma\right|= \begin{cases}\frac{q^{2}-q}{2} & \text { if } x \in \Sigma, \\ \frac{q^{2}+q}{2} & \text { if } x \in \mathrm{PG}\left(2, q^{2}\right) \backslash(S \cup \ell), \\ \frac{q^{2}}{2} & \text { if } x \in \ell \backslash\left\{U_{2}\right\}, \\ 0 & \text { if } x=U_{2} .\end{cases}
$$

Remark that $q(q-1) / 2$-regularity is the best we can achieve. Indeed, as we have already seen, every non-absolute point $v$ is adjacent to $q(q-1)$ other nonabsolute points and these neighbours come in pairs to form $q(q-1) / 2$ triangles with common vertex $v$, so $v$ can be adjacent to at most one vertex in each of these triangles. The fact that $\Sigma$ is $q(q-1) / 2$-regular implies that $v$ is adjacent to exactly one vertex in each of the triangles. In other words, if a triangle of $\operatorname{DUP}\left(q^{2}\right)$ contains a vertex of $\Sigma$, it contains another vertex of $\Sigma$. Thus we have shown the following result.

Corollary 4.10. Every triangle of $\operatorname{DUP}\left(q^{2}\right)$ has either 0 or 2 vertices in common with $\Sigma$.

This property allows us to show that the subgraph $\Sigma$ is maximal with the triangle-free property, i.e., we cannot add any vertex not in $\Sigma$ without creating a triangle.

Proposition 4.11. The graph $\Sigma$ is maximal with respect to the triangle-free property. 
Proof. Suppose we could add another vertex $v$. This vertex $v$ has at least one neighbour in $\Sigma$ as $v^{\perp}$ intersects any $\mathcal{U}_{\lambda}$ in at least one point. Therefore, consider a triangle $T$ containing $v$ and a vertex of $v^{\perp} \cap \Sigma$. From the previous Corollary, we know that the triangle $T$ actually has its third vertex in $\Sigma$ and hence we cannot add $v$ to $\Sigma$ without creating a triangle.

The next result shows that $\Sigma$ can be chosen in such a way that it has girth 5 . Note that the following construction asymptotically matches the best known lower bound on the maximum number of edges in a $n$-vertex graph with girth at least five [5].

Proposition 4.12. For $q$ an even prime power, there exists a $q(q-1) / 2$-regular graph on $q^{4} / 2$ vertices of girth 5 .

Proof. We can show the result for $q \leq 16$ using Magma [6], so suppose $q \geq 32$ for the remainder of the proof.

Taking into account Lemma 4.5, let $\Lambda=\{1 /(1+a) \mid a \in H\}$, where $H$ is an additive subgroup of $\mathbb{F}_{q}$ of order $q / 2$ not containing 1 . Let us consider a non-zero element $a \in H$ and let $\lambda_{1}=1 /(1+a) \in \Lambda$. Let $b \in H$, with $a \neq b$ such that $b$ is not a solution of none of the following equations:

$$
\begin{gathered}
X^{2}+(a+1) X+a^{3}=0, \quad X^{3}+a X+a(a+1)=0, \\
X^{2}+(a+1) X+a^{2}+a+1=0 .
\end{gathered}
$$

Since the union of the solutions of the equations (7) and (8) consists of at most 7 distinct elements of $\mathbb{F}_{q}$, we can always find such an element $b$ if $q \geq 32$. Let $P_{1}:=\left(1, x_{1}, 0\right) \in \mathcal{U}_{\lambda_{1}}$ and $P_{2}:=\left(1, x_{1}^{q}, 0\right)=P_{1}^{\perp} \cap \mathcal{U}_{\lambda_{1}}$ its unique neighbour on $\mathcal{U}_{\lambda_{1}}$. Take another point $Q_{1}:=\left(1, x_{1}^{q}, z\right) \in P_{1}^{\perp} \cap \mathcal{U}_{\lambda_{2}}$, with $\lambda_{2}=1 /(1+b) \in$ $\Lambda \backslash\left\{\lambda_{1}\right\}$. Hence $x_{1}+x_{1}^{q}=\lambda_{1}$ and $z^{q+1}=\lambda_{1}+\lambda_{2}$. Its unique neighbour on $\mathcal{U}_{\lambda_{2}}$ is $Q_{2}:=\left(1, x_{1}+\lambda_{1}+\lambda_{2}, z\right)=Q_{1}^{\perp} \cap \mathcal{U}_{\lambda_{2}}$. Then $R:=P_{2}^{\perp} \cap Q_{2}^{\perp}=\left(1, x_{1}, \lambda_{2} / z^{q}\right)$

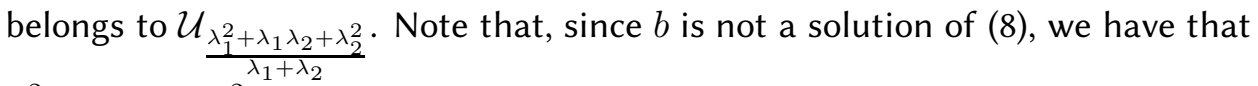
$\lambda_{1}^{2}+\lambda_{1} \lambda_{2}+\lambda_{2}^{2} \neq 0$. Hence $P_{1} P_{2} R Q_{2} Q_{1}$ is a cycle of length 5 in $\Sigma$ if and only if

$$
\frac{\lambda_{1}^{2}+\lambda_{1} \lambda_{2}+\lambda_{2}^{2}}{\lambda_{1}+\lambda_{2}} \in \Lambda
$$

On the other hand, a straightforward calculation shows that (9) holds true if and only if

$$
x:=\frac{a^{2} b+a b^{2}+a b+1}{a^{2}+b^{2}+a b+a+b+1} \in H .
$$


Note that $x \neq 1$, otherwise at least one among $a$ and $b$ should be 1 . Analogously to the proof of Lemma 4.5, view $\mathbb{F}_{q}$ as a vector space $V$ over $\mathbb{F}_{2}$. Since $b$ is not a solution of the equations (7), we have that the vector subspace of $V$ generated by $a, b$ and $x$ does not contain 1 . Therefore, we can find a hyperplane $H$ such that $a, b, x \in H$ and $1 \notin H$. This hyperplane corresponds to an additive subgroup of size $q / 2$, which concludes the proof.

\section{The Figueroa plane}

The finite Figueroa planes are non-Desarguesian projective planes of order $q^{3}$ for all prime powers $q>2$. These planes were constructed algebraically in 1982 by Figueroa [12], and Hering and Schaeffer [20], and synthetically in 1986 by Grundhöfer [16]. All Figueroa planes of finite square order possess a unitary polarity and hence admit unitals [11]. It is known that every polarity of $\mathcal{F}$ induces a polarity of $\mathrm{PG}\left(2, q^{3}\right)[19$, Theorem 4.2]. Vice versa, it can be seen that under certain assumptions, a polarity $\rho$ of the Desarguesian projective plane $\operatorname{PG}\left(2, q^{3}\right)$ is "inherited" and gives rise to a polarity $\rho^{\prime}$ of the Figueroa plane $\mathcal{F}$. In this section we show that, in the case of "inherited" polarities, a triangle-free induced subgraph of $\mathcal{G}\left(\mathrm{PG}\left(2, q^{3}\right), \rho\right)$ gives rise to a triangle-free induced subgraph of $\mathcal{G}\left(\mathcal{F}, \rho^{\prime}\right)$.

\subsection{Construction of Figueroa planes}

Let $\alpha$ be an order 3 collineation of the classical projective plane $\operatorname{PG}\left(2, q^{3}\right)$ of order $q^{3}$ over the finite field $\mathbb{F}_{q^{3}}$, where the fixed points of $\alpha$ constitute a subplane isomorphic to $\mathrm{PG}(2, q)$. The points and lines of $\mathrm{PG}\left(2, q^{3}\right)$ are partitioned into distinct types, as follows. A point $x$ of $\mathrm{PG}\left(2, q^{3}\right)$ belongs to $\mathcal{O}_{1}$ if $x^{\alpha}=x$, or to $\mathcal{O}_{2}$ if $x, x^{\alpha}, x^{\alpha^{2}}$ are distinct and on a line, or to $\mathcal{O}_{3}$ if $x, x^{\alpha}, x^{\alpha^{2}}$ are distinct and not on a line. Types of lines $\mathcal{L}_{1}, \mathcal{L}_{2}, \mathcal{L}_{3}$ of $\operatorname{PG}\left(2, q^{3}\right)$ are defined dually. Points of $\mathcal{O}_{1}$ and lines of $\mathcal{L}_{1}$ thus constitute a subplane $\mathcal{D}$ isomorphic to $\operatorname{PG}(2, q)$. If $x$ is a point of $\mathcal{O}_{2}$, then it is on the unique line of $\mathcal{L}_{1}$ containing $x, x^{\alpha}, x^{\alpha^{2}}$. Conversely, if a point is on a unique line of $\mathcal{L}_{1}$, then it belongs to $\mathcal{O}_{2}$ since a point of $\mathcal{O}_{1}$ is on $q+1$ lines of $\mathcal{L}_{1}$ and a point of $\mathcal{O}_{3}$ is on no line of $\mathcal{L}_{1}$. It follows that if (and only if) a point is on no line of $\mathcal{L}_{1}$, then it belongs to $\mathcal{O}_{3}$.

Let $\mu$ be an involutory bijection between the points of $\mathcal{O}_{3}$ and the lines of $\mathcal{L}_{3}$ given as follows: if $x \in \mathcal{O}_{3}$ and $\ell \in \mathcal{L}_{3}$, then $x^{\mu}=x^{\alpha} x^{\alpha^{2}}$, and $\ell^{\mu}=\ell^{\alpha} \cap \ell^{\alpha^{2}}$. The Figueroa plane $\mathcal{F}$ is obtained by the introduction of a new incidence between the set of points $\mathcal{P}$ and the set of lines $\mathcal{L}$ of $\mathrm{PG}\left(2, q^{3}\right)$, so that (viewing a line as a point set) the $q^{3}-q^{2}-q-2$ points of $\mathcal{O}_{3}$ on the line $\ell=x^{\alpha} x^{\alpha^{2}} \in \mathcal{L}_{3}$ distinct from $x^{\alpha}$ and $x^{\alpha^{2}}$ are replaced by other points of $\mathcal{O}_{3}$ to form a new line. More 
precisely, as a set of points, a line of $\operatorname{PG}\left(2, q^{3}\right)$ belonging to $\mathcal{L}_{1}$ or to $\mathcal{L}_{2}$ remains unchanged as a line in $\mathcal{F}$. As for a line $\ell=x^{\alpha} x^{\alpha^{2}} \in \mathcal{L}_{3}$ in $\operatorname{PG}\left(2, q^{3}\right)$, where $x \in \mathcal{O}_{3}$, let $y_{i}, 1 \leq i \leq q^{3}-q^{2}-q-2$, be the remaining points of $\mathcal{O}_{3}$ on $\ell$. Consider the pencil of lines of $\mathcal{L}_{3}$ on the point $x \in \mathcal{O}_{3}$. Other than $x x^{\alpha}$ and $x x^{\alpha^{2}}$, the remaining lines of $\mathcal{L}_{3}$ in the pencil are given by $z_{i} z_{i}^{\alpha}, 1 \leq i \leq q^{3}-q^{2}-q-2$, where each $z_{i}$ is a point of $\mathcal{O}_{3}$. Let $\ell_{\mathcal{F}}$ be the set of points obtained from $\ell$ by replacing each $y_{i}$ with $z^{\alpha^{2}}$. Then, $\ell_{\mathcal{F}}$ is the Figueroa line corresponding to the line $\ell$ of $\mathcal{L}_{3}$, see also [7]. Note that

$$
P \in \ell_{\mathcal{F}} \cap \mathcal{O}_{3} \text { if and only if } \ell^{\mu} \in P^{\mu} .
$$

We observe the following property.

Lemma 5.1. Let $\ell_{1}, \ell_{2} \in \mathcal{L}_{3}$ such that $\ell_{1} \cap \ell_{2} \in \mathcal{O}_{2}$. Then the line $\ell_{1}^{\mu} \ell_{2}^{\mu}$ belongs to $\mathcal{L}_{2}$.

Proof. Assume by contradiction that $\ell_{1}^{\mu} \ell_{2}^{\mu} \in \mathcal{L}_{3}$. Then $\left(\ell_{1}^{\mu} \ell_{2}^{\mu}\right)^{\mu} \in \mathcal{O}_{3}$. Since $\ell_{1}^{\mu} \in\left(\ell_{1}^{\mu} \ell_{2}^{\mu}\right)$ and $\ell_{2}^{\mu} \in\left(\ell_{1}^{\mu} \ell_{2}^{\mu}\right)$, we have that $\left(\ell_{1}^{\mu} \ell_{2}^{\mu}\right)^{\mu} \in\left(\ell_{1}\right)_{\mathcal{F}}$ and $\left(\ell_{1}^{\mu} \ell_{2}^{\mu}\right)^{\mu} \in\left(\ell_{2}\right)_{\mathcal{F}}$. On the other hand $\ell_{1} \cap \ell_{2}=\left(\ell_{1}\right)_{\mathcal{F}} \cap\left(\ell_{2}\right)_{\mathcal{F}}$, since $\ell_{1} \cap \ell_{2} \in \mathcal{O}_{2}$. It follows that $\left(\ell_{1}^{\mu} \ell_{2}^{\mu}\right)^{\mu}=\ell_{1} \cap \ell_{2}$, a contradiction.

\subsection{Polarities of $\mathcal{F}$}

Let $\rho$ be a polarity of $\operatorname{PG}\left(2, q^{3}\right)$ such that $\rho$ and $\alpha$ commute, i.e., $\rho \alpha=\alpha \rho$. Let $\mathcal{X}$ denote the set of $\rho$-absolute points.

Lemma 5.2. The following properties hold true:

1) $\mathcal{X}$ is preserved by $\alpha$,

2) the point $P \in \mathcal{O}_{i}$ if and only if $P^{\rho} \in \mathcal{L}_{i}$,

3) the line $\ell \in \mathcal{L}_{i}$ if and only if $\ell^{\rho} \in \mathcal{O}_{i}$,

4) if $P \in \mathcal{O}_{3}$, then $P^{\mu \rho}=P^{\rho \mu}$,

5) if $\ell \in \mathcal{L}_{3}$, then $\ell^{\mu \rho}=\ell^{\rho \mu}$.

Proof. Properties 1), 2) and 3) follow directly from the fact that the collineation $\alpha$ and the polarity $\rho$ commute. To prove 4 ), let $P$ be a point of $\mathcal{O}_{3}$, then

$$
P^{\mu \rho}=\left(P^{\alpha} P^{\alpha^{2}}\right)^{\rho}=P^{\alpha \rho} \cap P^{\alpha^{2} \rho}=P^{\rho \alpha} \cap P^{\rho \alpha^{2}}=P^{\rho \mu} .
$$

Property 5) follows similarly. 
Consider the following map $\rho_{\mathcal{F}}$ : for points and lines of $\mathcal{O}_{1}$ or $\mathcal{O}_{2}, \rho_{\mathcal{F}}=\rho$. For a point $x \in \mathcal{O}_{3}, x^{\rho_{\mathcal{F}}}=\left(x^{\rho}\right)_{\mathcal{F}}$, where $\left(x^{\rho}\right)_{\mathcal{F}}$ is the line of $\mathcal{F}$ corresponding to the line $x^{\rho} \in \mathcal{L}_{3}$ as described in the previous subsection. For a line $\ell \in \mathcal{L}_{3}$, let $\left(\ell_{\mathcal{F}}\right)^{\rho_{\mathcal{F}}}=\ell^{\rho}$. Since $\rho$ commutes with $\mu, \rho_{\mathcal{F}}$ is indeed a polarity of $\mathcal{F}$. Furthermore, if $x$ is a point of $\mathcal{O}_{3}$, then $x$ is $\rho_{\mathcal{F}}$-absolute if and only if $x^{\mu \rho} \in \mathcal{X}$. Hence, if we denote by $\mathcal{X}_{\mathcal{F}}$ the $\rho_{\mathcal{F}}$-absolute points, we have that

$$
\mathcal{X}_{\mathcal{F}}=\left(\mathcal{X} \cap \mathcal{O}_{1}\right) \cup\left(\mathcal{X} \cap \mathcal{O}_{2}\right) \cup\left\{x^{\mu \rho} \mid x \in \mathcal{X} \cap \mathcal{O}_{3}\right\}
$$

Since $\mu \rho$ is a bijection, the number of points of $\mathcal{O}_{3}$ which are $\rho_{\mathcal{F}}$-absolute equals the number of points of $\mathcal{O}_{3}$ which are $\rho$-absolute. Thus, the number of absolute points of $\rho_{\mathcal{F}}$ is the same as that of $\rho$.

A stronger result than Lemma 5.2 has been proved by Hamilton in his Ph.D thesis.

Theorem 5.3. [19, Theorem 4.2] Every polarity of the Figueroa plane $\mathcal{F}$ induces a polarity of its Desarguesian subplane $\mathcal{D}$ and vice versa.

Note that, since a polarity of $\mathcal{D}$ extends to a polarity of $\operatorname{PG}\left(2, q^{3}\right)$, it easily follows that a polarity of $\mathcal{F}$ induces a polarity of $\mathrm{PG}\left(2, q^{3}\right)$.

We end this section by considering the self-polar triangles with respect to polarities of $\mathcal{F}$.

Lemma 5.4. Let $T$ be a triangle containing at least two points of $\mathcal{O}_{1} \cup \mathcal{O}_{2}$. Then $T$ is self polar with respect to $\rho$ if and only if $T$ is self-polar with respect to $\rho_{\mathcal{F}}$.

Proof. Let $T=\left\{P_{1}, P_{2}, P_{2}\right\}$, where $P_{1}, P_{2} \in T \cap\left(\mathcal{O}_{1} \cup \mathcal{O}_{2}\right)$. Then $P_{1}^{\rho}=P_{1}^{\rho_{\mathcal{F}}}=$ $P_{2} P_{3}$ and $P_{2}^{\rho}=P_{2}^{\rho_{\mathcal{F}}}=P_{1} P_{3}$. On the other hand, $P_{3}^{\rho}=P_{1} P_{2}$ if and only if $P_{3}=P_{1}^{\rho} \cap P_{2}^{\rho}=P_{1}^{\rho_{\mathcal{F}}} \cap P_{2}^{\rho_{\mathcal{F}}}$ if and only if $P_{3}^{\rho_{\mathcal{F}}}=P_{1} P_{2}$, as required.

Remark 5.5. Note that if a triangle $T$ has at least one of its points in $\mathcal{O}_{1}$, then $T$ is contained in $\mathcal{O}_{1} \cup \mathcal{O}_{2}$, while if two of its points are in $\mathcal{O}_{1}$, then its third point will belong to $\mathcal{O}_{1}$ as well.

Lemma 5.6. There is a bijection between the self-polar triangles of $\mathrm{PG}\left(2, q^{3}\right)$ with respect to $\rho$ and the self-polar triangles of $\mathcal{F}$ with respect to $\rho_{\mathcal{F}}$.

Proof. Taking into account Lemma 5.4 and Remark 5.5, we can consider the selfpolar triangles containing no point of $\mathcal{O}_{1}$ and at most one point of $\mathcal{O}_{2}$. Let $T=$ $\left\{P_{1}, P_{2}, P_{3}\right\}$ be a self-polar triangle of $\mathrm{PG}\left(2, q^{3}\right)$ with respect to $\rho$. Let $P_{i}^{\rho}=\ell_{i}$, that is, $\ell_{1}=P_{2} P_{3}, \ell_{2}=P_{1} P_{3}, \ell_{3}=P_{1} P_{2}$. Assume first that $T \subseteq \mathcal{O}_{3}$. We show 
that $T$ is a self-polar with respect to $\rho$ if and only if $T_{\mathcal{F}}=\left\{\ell_{1}^{\mu}, \ell_{2}^{\mu}, \ell_{3}^{\mu}\right\}$ is a self polar triangle with respect to $\rho_{\mathcal{F}}$. Indeed,

$$
\begin{aligned}
\ell_{1}^{\mu}=\ell_{2}^{\mu \rho_{\mathcal{F}}} \cap \ell_{3}^{\mu \rho_{\mathcal{F}}} & \Longleftrightarrow \ell_{2}^{\mu}, \ell_{3}^{\mu} \in \ell_{1}^{\mu \rho_{\mathcal{F}}}=\left(\ell_{1}^{\mu \rho}\right)_{\mathcal{F}} \\
& \Longleftrightarrow\left(\ell_{1}^{\mu \rho}\right)^{\mu} \in\left(\ell_{2}^{\mu}\right)^{\mu},\left(\ell_{3}^{\mu}\right)^{\mu} \\
& \Longleftrightarrow \ell_{1}^{\rho} \in \ell_{2}, \ell_{3} \\
& \Longleftrightarrow P_{1} \in P_{2}^{\rho}, P_{3}^{\rho} \\
& \Longleftrightarrow P_{1}=P_{2}^{\rho} \cap P_{3}^{\rho},
\end{aligned}
$$

and similarly for any permutation of the indices.

On the other hand, if $P_{1} \in \mathcal{O}_{2}$ and $P_{2}, P_{3} \in \mathcal{O}_{3}$, let $P=\ell_{2}^{\mu \rho} \cap \ell_{3}^{\mu \rho}=$ $\left(\ell_{2}^{\mu} \ell_{3}^{\mu}\right)^{\rho}=P_{2}^{\mu} \cap P_{3}^{\mu}$. Then, taking into account Lemma 5.1 and Lemma 5.2, we have that $P \in \mathcal{O}_{2}$. Moreover, $T$ is a self-polar triangle with respect to $\rho$ if and only $T_{\mathcal{F}}=\left\{\ell_{2}^{\mu}, \ell_{3}^{\mu}, P\right\}$ is a self polar triangle with respect to $\rho_{\mathcal{F}}$. Indeed, a similar argument as used above gives $P_{2}=P_{1}^{\rho} \cap P_{3}^{\rho}$ if and only if $\ell_{2}^{\mu}=\ell_{3}^{\mu \rho_{\mathcal{F}}} \cap P^{\rho_{\mathcal{F}}}$ and $P_{3}=P_{1}^{\rho} \cap P_{2}^{\rho}$ if and only if $\ell_{3}^{\mu}=\ell_{2}^{\mu \rho_{\mathcal{F}}} \cap P^{\rho_{\mathcal{F}}}$. Moreover, since $P \in \ell_{i}^{\mu \rho} \cap \mathcal{O}_{2} \subset$ $\ell_{i}^{\mu \rho} \cap\left(\ell_{i}^{\mu \rho}\right)_{\mathcal{F}}, i=2,3$, it follows that $P \in\left(\ell_{i}^{\mu \rho}\right)_{\mathcal{F}}=\ell_{i}^{\mu \rho_{\mathcal{F}}}$.

Theorem 5.7. Let $\mathcal{Z}$ be a triangle-free set consisting of non-absolute points with respect to $\rho$, then

$$
\mathcal{Z}_{\mathcal{F}}=\left(\mathcal{Z} \cap \mathcal{O}_{1}\right) \cup\left\{x^{\mu \rho} \mid x \in \mathcal{Z} \cap \mathcal{O}_{3}\right\}
$$

is a triangle-free set consisting of non-absolute points with respect to $\rho_{\mathcal{F}}$.

Proof. Assume by contradiction that there exists a self-polar triangle with respect to $\rho_{\mathcal{F}}$, say $T_{\mathcal{F}}$, contained in $\mathcal{Z}_{\mathcal{F}}$, then necessarily $T_{\mathcal{F}}$ is contained in $\mathcal{O}_{3}$. If $T_{\mathcal{F}}=$ $\left\{P_{1}^{\rho \mu}, P_{2}^{\rho \mu}, P_{3}^{\rho \mu}\right\}$, then it follows that $T=\left\{P_{1}, P_{2}, P_{3}\right\}$ is a self-polar triangle with respect to $\rho$ contained in $\mathcal{Z}$, a contradiction.

Finally, taking into account Theorem 1.3 and Theorem 5.7, we have the following.

Corollary 5.8. Let $\rho$ be a unitary polarity of $\mathrm{PG}\left(2, q^{6}\right)$, $q$ even, and let $\alpha$ be an order 3 collineation of $\mathrm{PG}\left(2, q^{6}\right)$ fixing a subplane $\mathcal{D} \cong \mathrm{PG}\left(2, q^{2}\right)$ pointwise, such that $\rho$ and $\alpha$ commute. Then, there exists a set of non-absolute vertices of $\mathcal{G}\left(\mathcal{F}, \rho_{\mathcal{F}}\right)$ inducing a triangle-free subgraph of size $\frac{q^{12}-q^{4}\left(q^{4}-1\right)\left(q^{2}+1\right)}{2}$.

Proof. From Theorem 1.3, there exists a set $\mathcal{Z}$ of non-absolute vertices of $\mathcal{G}\left(\mathrm{PG}\left(2, q^{6}\right), \rho\right)$ inducing a triangle-free subgraph of size $q^{12} / 2$. From Theorem 5.7, the set $\mathcal{Z}_{\mathcal{F}}$ is a triangle-free set consisting of non-absolute points with respect to $\rho_{\mathcal{F}}$, where 
$\left|\mathcal{Z}_{\mathcal{F}}\right|=\left|\mathcal{Z} \backslash \mathcal{O}_{2}\right|$. We will count the number of points of $\mathcal{Z} \cap \mathcal{O}_{2}$, which we have to remove, by inspecting the $q^{4}+q^{2}+1$ lines of $\mathcal{L}_{1}$. Recall that these lines only contain points of $\mathcal{O}_{1}$ and $\mathcal{O}_{2}$. As every point of $\mathcal{O}_{2}$ lies on exactly one line of $\mathcal{L}_{1}$, we will find every point of $\mathcal{Z} \cap \mathcal{O}_{2}$ once.

By construction, $\mathcal{Z}$ is the union of $q^{3} / 2$ Hermitian curves of $\operatorname{PG}\left(2, q^{6}\right)$ pairwise meeting in a point $U_{2}$ of $\mathcal{D}$ and having the same tangent line $\ell$ at $U_{2}$, with their common point $U_{2}$ deleted. Among these Hermitian curves there are $q / 2$ meeting $\mathcal{D}$ in a Hermitian curve of $\mathrm{PG}\left(2, q^{2}\right)$. One can see this by using the vector space representation of $\mathbb{F}_{q^{3}}$ over $\mathbb{F}_{2}$ : the $q^{3} / 2$ Hermitian curves are parametrized by elements of $\mathbb{F}_{q^{3}}$, which form a hyperplane. As $\mathbb{F}_{q}$ is a subspace of $\mathbb{F}_{q^{3}}$ not properly contained in the hyperplane, as it does not contain the element $1 \in \mathbb{F}_{q}$, this means that it intersects $\mathbb{F}_{q}$ in $q / 2$ points, which parametrize the $q / 2$ Hermitian curves in $\mathcal{D}$. It follows that for a line $r \in \mathcal{L}_{1}$, we can compute $\left|r \cap\left(\mathcal{Z} \cap \mathcal{O}_{2}\right)\right|=|r \cap \mathcal{Z}|-\left|r \cap \mathcal{Z} \cap \mathcal{O}_{1}\right|$ using the intersection properties as stated in Corollary 4.9 in both $\mathrm{PG}\left(2, q^{6}\right)$ and $\mathcal{D}=\mathrm{PG}\left(2, q^{2}\right)$ respectively. Therefore, if $r$ is a line of $\mathcal{O}_{1}$, then

$$
|r \cap \mathcal{Z}|-\left|r \cap \mathcal{Z} \cap \mathcal{O}_{1}\right|= \begin{cases}\frac{q^{6}-q^{3}}{2}-\frac{q^{2}-q}{2} & \text { if } U_{2} \notin r \text { and } r^{\rho} \in \mathcal{Z}, \\ \frac{q^{6}+q^{3}}{2}-\frac{q^{2}+q}{2} & \text { if } U_{2} \notin r \text { and } r^{\rho} \notin \mathcal{Z}, \\ \frac{q^{6}}{2}-\frac{q^{2}}{2} & \text { if } U_{2} \in r \neq \ell, \\ 0 & \text { if } r=\ell .\end{cases}
$$

The number of lines corresponding to each case is respectively $q^{4} / 2, q^{4} / 2, q^{2}$ and 1. Summing up over all these lines, we obtain the number $\left|\mathcal{Z} \cap \mathcal{O}_{2}\right|$ which we had to subtract from $q^{12} / 2$ to obtain the result.

\section{Conclusion and open problems}

In [24] the following question was posed.

Question 6.1. Given a finite projective plane $\pi$ of order $q$ and a polarity $\perp$, is it possible to find a triangle-free subgraph of the polarity graph of size $\frac{1}{2} q^{2}+o\left(q^{2}\right)$ ?

When $\pi=\mathrm{PG}(2, q)$, this question has been almost completely resolved. Depending on the parity of $q$ and the type of $\perp$, there are four possibilities, shown in the table below. 


\begin{tabular}{|c|c|c|}
\hline prime power $q$ & type & answer \\
\hline even & pseudo & yes [26] \\
odd & orthogonal & yes [28] \\
even square & unitary & yes \\
odd square & unitary & $?$ \\
\hline
\end{tabular}

Starting from Question 6.1, we can state three open problems, ranked in what we believe to be increasing difficulty.

Open problem 1. Show that there exists a triangle-free induced subgraph of $\operatorname{DUP}\left(q^{2}\right), q$ odd, of size $\frac{1}{2} q^{4}+o\left(q^{4}\right)$.

In Section 4.1 we mention the existence of a triangle-free induced subgraph of DUP $\left(q^{2}\right), q$ odd, of size $q^{4} / 3+o\left(q^{4}\right)$. Other ideas will be needed to find larger triangle-free subgraphs.

Open problem 2 (Conjecture 1 in [27]). Prove or disprove that in case 2, i.e. $\pi=\mathrm{PG}(2, q)$ and $\perp$ is an orthogonal polarity, Parsons' examples are the largest. If true, is it possible to show that they are the unique triangle-free induced subgraphs of this size?

As shown by Loucks and Timmons, this can only be true when $q$ is large enough. Using a computer search, they found larger examples for $q=5,7,9,13$.

Open problem 3. What if $\pi$ is not the Desarguesian projective plane $\operatorname{PG}(2, q)$ ? Can we still answer Question 6.1 in the affirmative?

In the case when $\pi$ is the Figueroa plane and the unitary polarity is inherited, we showed that the answer is indeed yes. In fact, one can do this for any inherited polarity by Theorem 5.7, but for this article, we restrict ourselves to the unitary case.

Lastly, remark that there exist projective planes of order $q$ and polarities where the size of the set of absolute points does not belong to $\{q+1, q \sqrt{q}+1\}$, see [22, Chapter XII], [30].

Acknowledgment. We thank the referees for their valuable comments which improved the quality of this paper. We also thank B. Csajbók for the proofs of Lemma 4.4 and Theorem 4.7 and A. Bishnoi and A. Potukuchi for the proof of the prime case independently. 


\section{References}

[1] R. Baer, Polarities in finite projective planes, Bull. Amer. Math. Soc., 52:7793, 1946.

[2] S. Barwick, G. Ebert, Unitals in projective planes, Springer Monographs in Mathematics, Springer New York, 2008.

[3] A. Bishnoi, S. Mattheus, J. Schillewaert, Minimal multiple blocking sets, https://arxiv.org/abs/1703.07843, 2017.

[4] A. Bishnoi, A. Potukuchi, Personal communication.

[5] N.H. Bong, Properties and structures in extremal graphs, PhD thesis, University of Newcastle, Australia, 2017.

[6] W. Bosma, J. Cannon, C. Playoust, The Magma algebra system. I. The user language. Computational algebra and number theory, J. Symbolic Comput., 24:235-265, 1997.

[7] J.M.N. Brown, Some partitions in Figueroa planes, Note Math. 29:33-44, 2009.

[8] R. Calderbank, W.M. Kantor, The geometry of two-weight codes, Bull. London Math. Soc., 18:97-122, 1986.

[9] B. Csajbók, Personal communication.

[10] B. De Bruyn, H. Suzuki, Intriguing sets of vertices of regular graphs, Graphs Combin., 26:629-646, 2010.

[11] M. De Resmini, N. Hamilton, Hyperovals and unitals in Figueroa planes, Eur. J. Combin., 19:215-220, 1998.

[12] R. Figueroa, A family of not $(V, l)$-transitive projective planes of order $q^{3}$, $q \not \equiv 1(\bmod 3)$ and $q>2$, Math. Z., 181:471-479, 1982.

[13] Z. Füredi, Graphs without Quadrilaterals, J. Combin. Theory Ser. B, 34:187190, 1983.

[14] Z. Füredi, On the Number of Edges of Quadrilateral-Free Graphs, J. Combin. Theory Ser. B, 68:1-6, 1996.

[15] C.D. Godsil, M.W. Newman, Eigenvalue bounds for independent sets, J. Combin. Theory Ser. B, 98:721-734, 2008. 
[16] T. Grundhöfer, A synthetic construction of the Figueroa planes, J. Geom., 26, 191-201, 1986.

[17] W.H. Haemers, Eigenvalue techniques in design and graph theory, PhD thesis, University of Eindhoven, 1979.

[18] W.H. Haemers, Interlacing Eigenvalues and Graphs, Linear Algebra Appl., 226/228:593-616, 1995.

[19] N. Hamilton, Maximal Arcs in Finite Projective Planes and Associated Structures in Projective Spaces, PhD thesis, University of Western Australia, 1995.

[20] Ch., Hering, H.J. Schaeffer, On the new projective planes of R. Figueroa, In: Jungnickel, D. et al. (eds.) Combinatorial Theory. Proc. Schloss Rauischholzhausen, 1982, pp. 187-190. Springer, Berlin, 1982.

[21] J.W.P. Hirschfeld, Projective Geometries over Finite Fields, Oxford Mathematical Monographs, Oxford Science Publications, The Clarendon Press, Oxford University Press, New York, 1998.

[22] D. Hughes, F. Piper, Projective planes, Graduate Texts in Mathematics, Springer-Verlag New York-Berlin, 1973.

[23] F. Lazebnik, J. Verstraete, On hypergraphs of girth five, Electron. J. Combin., 10:25, 2003.

[24] J. Loucks, C. Timmons, Triangle-free induced subgraphs of polarity graphs, https://arxiv.org/abs/1703.06347, 2017.

[25] B. Lund, S. Saraf. Incidence bounds for block designs, SIAM J. Discrete Math., 30:1997-2010, 2016.

[26] S. Mattheus, F. Pavese, L. Storme, On the independence number of graphs related to a polarity, https://arxiv.org/pdf/1704.00487, 2017.

[27] D. Mubayi, J. Williford, On the independence number of the Erdős-Rényi and projective norm graphs and a related hypergraph, J. Graph Theory, 56:113-127, 2007.

[28] T.D. Parsons, Graphs from projective planes, Aequationes Math., 14:167-189, 1976.

[29] T. Penttila, G. Royle, Sets of type $(m, n)$ in the Affine and Projective Planes of Order Nine, Des. Codes Cryptogr., 6:229-245, 1995. 
[30] F. Piper, Polarities in the Hughes plane, Bull. London Math. Soc. 2:209-213, 1970.

[31] S. D. Winter, J. Schillewaert, and J. Verstraete, Large incidence-free sets in geometries, Electron. J. Combin, 19:24, 2012.

[32] P. Xing, M. Tait, C. Timmons, On the chromatic number of the Erdős-Rényi orthogonal polarity graph, Electron. J. Combin., 22:2.21, 2015. 